Check for updates

Cite this: RSC Adv., 2019, 9, 26831

Received 6th July 2019

Accepted 21st August 2019

DOI: 10.1039/c9ra05134a

rsc.li/rsc-advances

\section{Pressure-induced enhancement of thermoelectric power factor in pristine and hole-doped SnSe crystals}

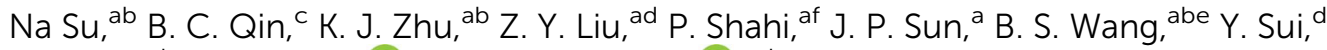

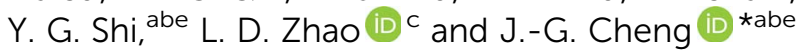

We evaluate the influence of pressure on the thermoelectric power factors $\mathrm{PF} \equiv S^{2} \sigma$ of pristine and $\mathrm{Na}$ doped SnSe crystals by measuring their electrical conductivity $\sigma(T)$ and Seebeck coefficient $S(T)$ up to $\sim 22$ kbar with a self-clamped piston-cylinder cell. For both cases, $\sigma(T)$ is enhanced while $S(T)$ reduced with increasing pressure as expected, but their imbalanced variations lead to a monotonic enhancement of PF under pressure. For pristine SnSe, $\sigma(290 \mathrm{~K})$ increases by $\sim 4$ times from $\sim 10.1$ to $38 \mathrm{~S} \mathrm{~cm}^{-1}$, while $S(290 \mathrm{~K})$ decreases by only $\sim 12 \%$ from 474 to $415 \mu \mathrm{V} \mathrm{K}-1$, leading to about three-fold enhancement of PF from 2.24 to $6.61 \mu \mathrm{W} \mathrm{cm}{ }^{-1} \mathrm{~K}^{-2}$, which is very close to the optimal value of SnSe above the structural transition at $\sim 800 \mathrm{~K}$ at ambient pressure. In comparison, the PF of Na-doped SnSe at $290 \mathrm{~K}$ is enhanced moderately by $\sim 30 \%$ up to $20 \mathrm{kbar}$. In contrast, the PF of isostructural black phosphorus with a simple band structure was found to decrease under pressure. The comparison with black phosphorus indicates that the multi-valley valence band structure of SnSe is beneficial for the enhancement of PF by retaining a large Seebeck coefficient under pressure. Our results also provide experimental confirmation on the previous theoretical prediction that high pressure can be used to optimize the thermoelectric efficiency of SnSe.

\section{Introduction}

Thermoelectric (TE) materials have attracted considerable attention in recent years because they can directly convert waste heat into useful electrical energy. ${ }^{1}$ The conversion efficiency of TE materials is characterized by the dimensionless figure of merit, $Z T \equiv S^{2} \sigma T / \kappa$, where $S$ is the Seebeck coefficient, $\sigma$ is the electrical conductivity, $\kappa$ is the thermal conductivity, and $T$ is the absolute temperature. The $Z T$ value of TE materials should be improved to $\geq 3$ for a widespread usage economically, whereas the most efficient TE materials so far have $Z T$ values around 2. Thus, much effort has been devoted to develop new TE materials to achieve a high $Z T .^{2-4}$

One important recent development is the discovery of exceptional TE performance in SnSe crystal with a high $Z T$ value of $2.6 \pm 0.3$ at $923 \mathrm{~K}$ along the $b$-axis, which has been attributed

\footnotetext{
${ }^{a}$ Beijing National Laboratory for Condensed Matter Physics, Institute of Physics, Chinese Academy of Sciences, Beijing 100190, China. E-mail: jgcheng@iphy.ac.cn

${ }^{b}$ School of Physical Sciences, University of Chinese Academy of Sciences, Beijing 100190, China

'School of Materials Science and Engineering, Beihang University, Beijing 100191, China

${ }^{d}$ School of Physics, Harbin Institute of Technology, Harbin 15001, China

${ }^{e}$ Songshan Lake Materials Laboratory, Dongguan, Guangdong 523808, China

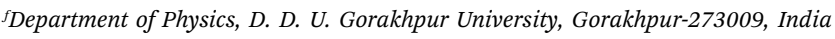

to an intrinsically ultralow $\kappa$ as a result of strong anharmonic bonding. ${ }^{5,6}$ At ambient condition, SnSe adopts a layered orthorhombic crystal structure with space group Pnma (no. 62), inset of Fig. 1, featured by corrugated two-atom-thick SnSe slabs within the $b c$ plane. Upon heating, SnSe undergoes a displacive structural transition to a higher symmetry $\mathrm{Cmcm}$ phase at about 750-800 K. Although the TE performance of SnSe crystal is superior above $800 \mathrm{~K}$ in the high- $\mathrm{T} \mathrm{Cmcm}$ phase, its performance in the low- $T$ Pnma phase is marginal, e.g. $Z T \sim 0.12$ at room temperature. ${ }^{5}$ Such characteristics limit its practical usage over a wide temperature range. Therefore, there have been extensive follow-up investigations on SnSe and related materials aiming to further improve their TE performances through various approaches. ${ }^{7-17}$ Among them, the most commonly used one is the chemical substitutions for $\mathrm{Sn} / \mathrm{Se}$ or introducing vacancies.

Although the carrier doping approach is very effective on improving the TE properties of SnSe, to understand the underlying mechanisms is complicated by the concurrent factors such as carrier centration, lattice disorders or defects, etc. In comparison, Zhang et al., ${ }^{18}$ by employing a computational method, proposed that the application of high pressure can intrinsically enhance the TE properties of SnSe in the low- $T$ Pnma phase due to a significant enhancement of power factor $\mathrm{PF} \equiv S^{2} \sigma$. Because $S$ and $\sigma$ are two anti-correlated transport quantities that are closely related with each other, the 

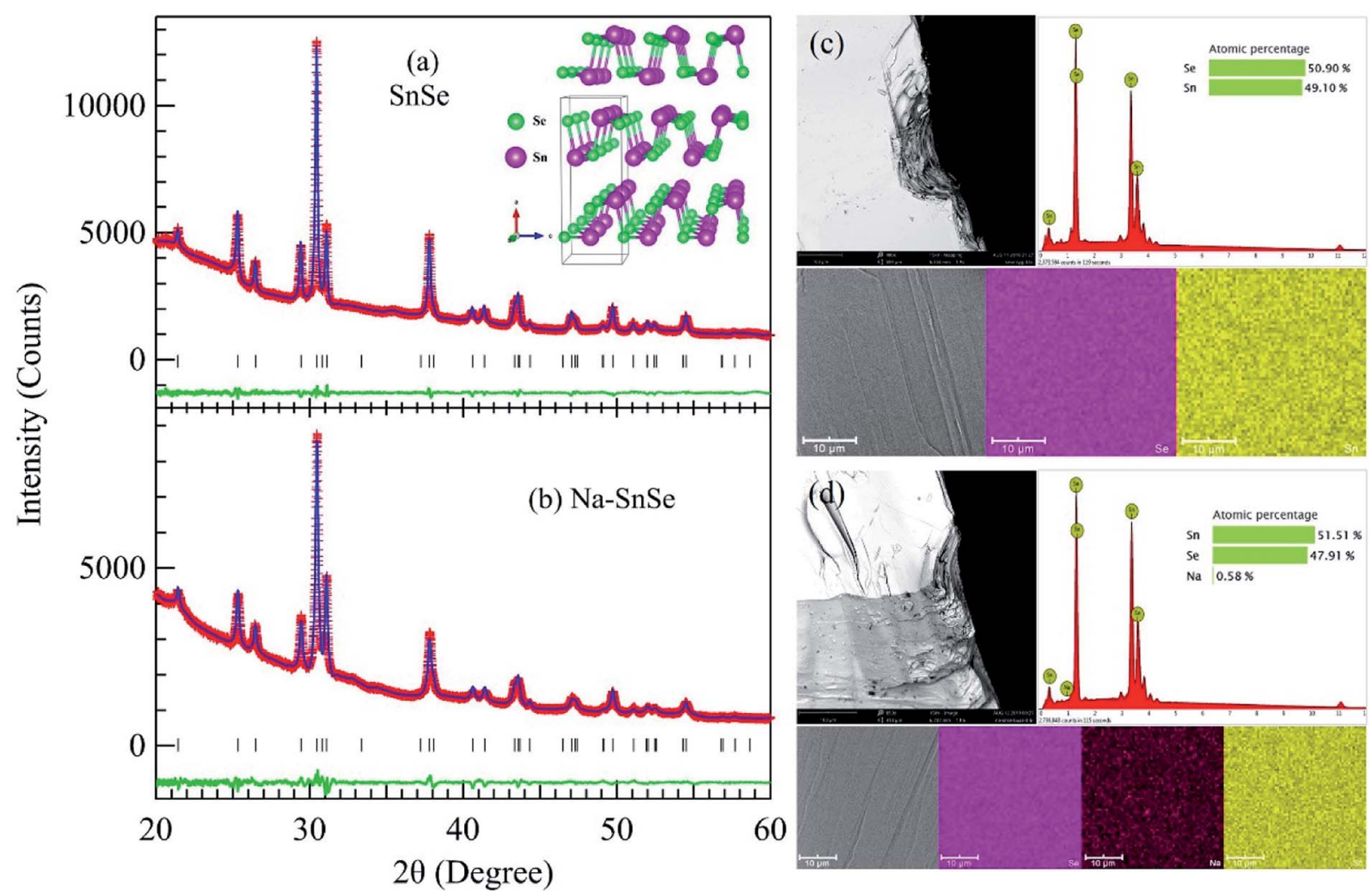

Fig. 1 Powder XRD patterns of (a) pristine and (b) Na-doped SnSe after Rietveld refinements, images of SEM, EDS, and element distribution maps for (c) pristine and (d) Na-doped SnSe. Inset of (a) shows the crystal structure of SnSe at the low-temperature Pnma phase.

enhancement of PF would suggest a peculiar electronic band structure that promotes imbalanced contributions to these two transport properties under pressure. In particular, the multiple electronic valence bands in SnSe have been argued to be responsible for the ultra-high PF. ${ }^{6}$ An experimental verification for this prediction can thus shed more light on the mechanism of excellent TE performance in SnSe, in addition to providing other means to optimize its performance.

To this end, we have performed a simultaneous measurement of $\sigma(T)$ and $S(T)$ on SnSe crystals under various pressures up to 22 kbar with a piston cylinder type high-pressure cell. Our results confirm the prediction by Zhang et al., ${ }^{18}$ and reveal a three-fold enhancement of PF from ambient pressure to 22 kbar, without showing a trend of saturation under higher pressures. We also observe a moderate enhancement of PF on Na-doped SnSe crystal under pressure, whereas the PF of isostructural black phosphorus with a single band is found to decrease in the similar pressure range. The comparison with black phosphorous underlines the importance of multiple valley electronic band structure in retaining a large Seebeck coefficient so as to boost the PF of SnSe under pressure.

\section{Experimental}

\section{Sample preparation and characterizations}

Crystals of pristine and Na-doped SnSe were prepared by the vertical Bridgman method. High-purity elemental constitutes of $\mathrm{Sn}, \mathrm{Se}$, and $\mathrm{Na}$ were weighted in a stoichiometric ratio of SnSe and $\mathrm{Sn}_{0.985} \mathrm{Na}_{0.015} \mathrm{Se}$. The starting chemicals were loaded into carbon-coated conical silicon tubes under a glove box with nitrogen atmosphere. The tubes were evacuated and flamesealed under pressure of $\sim 10^{-3} \mathrm{~Pa}$. The outer tubes were used to prevent samples from oxidation because the inner tubes could break caused by high-temperature phase transition. The tubes were then loaded into vertical furnace, heated up to 1313 $\mathrm{K}$ at rate of $10 \mathrm{~K} \mathrm{~min}^{-1}$ and soaked for $10 \mathrm{~h}$, subsequently cooled to $1073 \mathrm{~K}$ at a slow rate of $1 \mathrm{~K} \mathrm{~h}^{-1}$ and then furnace cooled to room temperature. Finally, the pristine and Na-doped SnSe crystals with diameter of $\sim 12 \mathrm{~mm}$ and length of $\sim 30 \mathrm{~mm}$ were obtained. Details about the sample preparation and characterizations can also be found elsewhere. ${ }^{5,6}$

Phase purity of the studied crystals has been verified at room temperature with powder X-ray diffraction (XRD) by using $\mathrm{Cu}-$ $\mathrm{K} \alpha$ radiation $(\lambda=1.5406 \AA)$. Fig. 1 (a) and (b) displays the XRD patterns after Rietveld refinements for pristine and Na-doped SnSe, respectively. No secondary phase is detectable for both samples, signaling a high purity for the studied crystals. The refinements were performed in the orthorhombic structure defined in space group Pnma (no. 62) with Sn and Se atoms both placed at $4 \mathrm{c}(x, 1 / 4, z)$ sites. As illustrated in Fig. 1(a) and (b), the refinement converged well with small reliability factors, i.e. $R_{\mathrm{p}}=$ $1.49 \%, R_{\mathrm{wp}}=1.96 \%$ for SnSe, and $R_{\mathrm{p}}=1.53 \%, R_{\mathrm{wp}}=2.06 \%$ for Na-SnSe, respectively. The obtained lattice parameters, $a=$ 11.4913(4) $\mathrm{\AA}, b=4.1504(1) \AA, c=4.4411(2) \AA$, and $V=211.81(1)$ $\AA^{3}$ for SnSe, and $a=11.4983(5) \AA, b=4.1500(2) \AA, c=4.4376(3)$ $\AA$, and $V=211.75(2) \AA^{3}$ for Na-SnSe, are close to those reported 
(a)

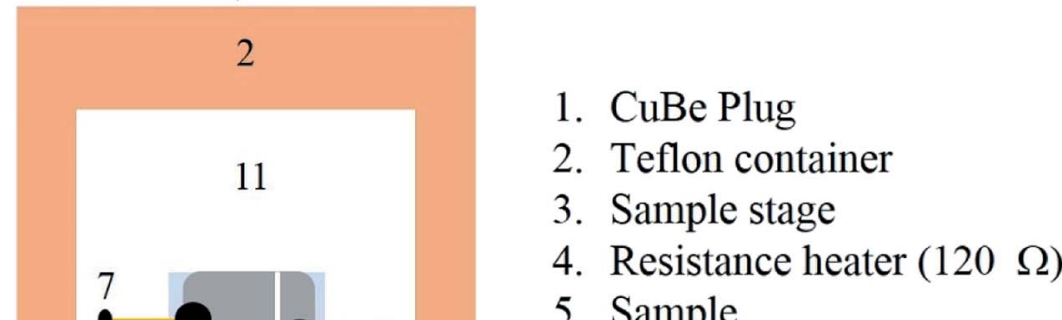

5. Sample

6-9. Electric leads for $\mathrm{V}_{\text {hot }} / \mathrm{I}+, \mathrm{V}_{\text {cold }} / \mathrm{I}-, \mathrm{V}+$, and $\mathrm{V}-$

10. AuFe/chromel differential thermocouple

11. Pressure transmitting medium (Daphne 7373)

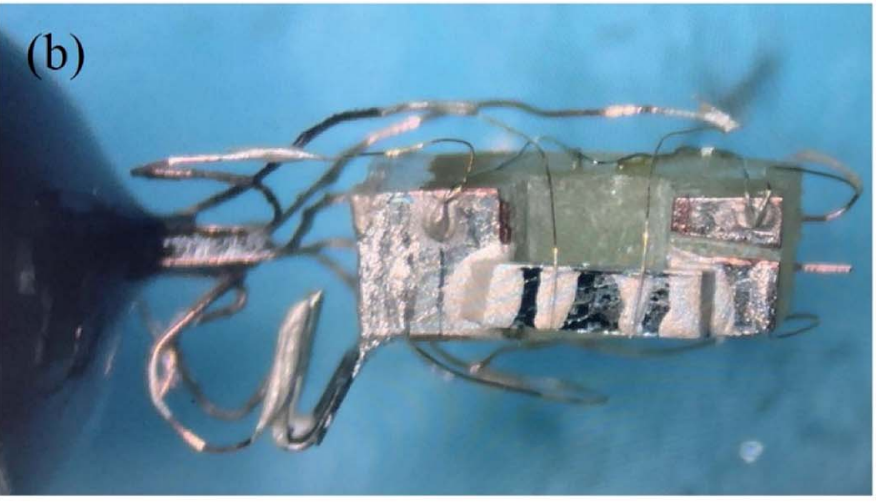

Fig. 2 (a) A schematic drawing of the setup for high-pressure Seebeck coefficient and resistivity measurements inside a clamp-type pistoncylinder cell. (b) A picture of the sample assembly inside the Teflon container.

previously., ${ }^{5,6}$ It also reveals that the influence of small $\mathrm{Na}$ doping on the lattice parameters of SnSe is negligible.

The morphology and the chemical composition for the studied crystals are further characterized by a Phenom scanning electron microscope (SEM) equipped with energy-dispersive Xray spectroscopy (EDS). The results for pristine and Na-doped SnSe are summarized in Fig. 1(c) and (d), respectively. Flat surfaces with layered structure for both samples can be viewed clearly from the SEM images in Fig. 1(c) and (d). The EDS maps confirm a uniform element distribution in both samples, and the statistical EDS analyses reveal a slight off-stoichiometry for the pristine and Na-doped SnSe with Sn vacancies.

\section{High-pressure setup for TE property measurements}

The crystals were oriented with the Laue back diffraction method and cut into a rectangular shape with the longest dimension along the orthorhombic $b$-axis. Temperature dependencies of Seebeck coefficient, $S(T)$, and electrical conductivity, $\sigma(T)$, under various pressures were measured simultaneous in a self-clamped piston-cylinder cell (PCC). A standard four-probe method was used to measure $\sigma(T)$ with the current applied along the $b$-axis; while a steady-state method was employed to measure $S(T)$ in a home-made setup. ${ }^{19} \mathrm{~A}$ schematic drawing of the setup inside the PCC is shown in Fig. 2(a), and a picture of the sample assembly inside the Teflon container is given in Fig. 2(b). The temperature gradient $\Delta T / T \sim$ $1 \%$ for $S(T)$ measurements was created across the sample via applying electrical current to a resistance heater $\left(\begin{array}{ll}\sim 20 & \Omega\end{array}\right)$ attached to one end of the sample stage. The absolute value of $\Delta T$ at each temperature was recorded with an AuFe/chromel differential thermocouple with an accuracy of $0.2 \%$. The precision for the $S(T)$ measurement is about $0.5 \mu \mathrm{V} \mathrm{K}{ }^{-1}$. The electric leads to measure the voltage difference $\Delta V=V_{\text {hot }}-V_{\text {cold }}$ of Seebeck coefficient also serve as the current leads, $I^{+}$and $I$, for resistivity measurements, during which no temperature gradient was created. Daphne 7373 was used as the pressure transmitting medium. The pressure inside the pressure cell was determined at room temperature by using the manganin wire resistance gauge. 

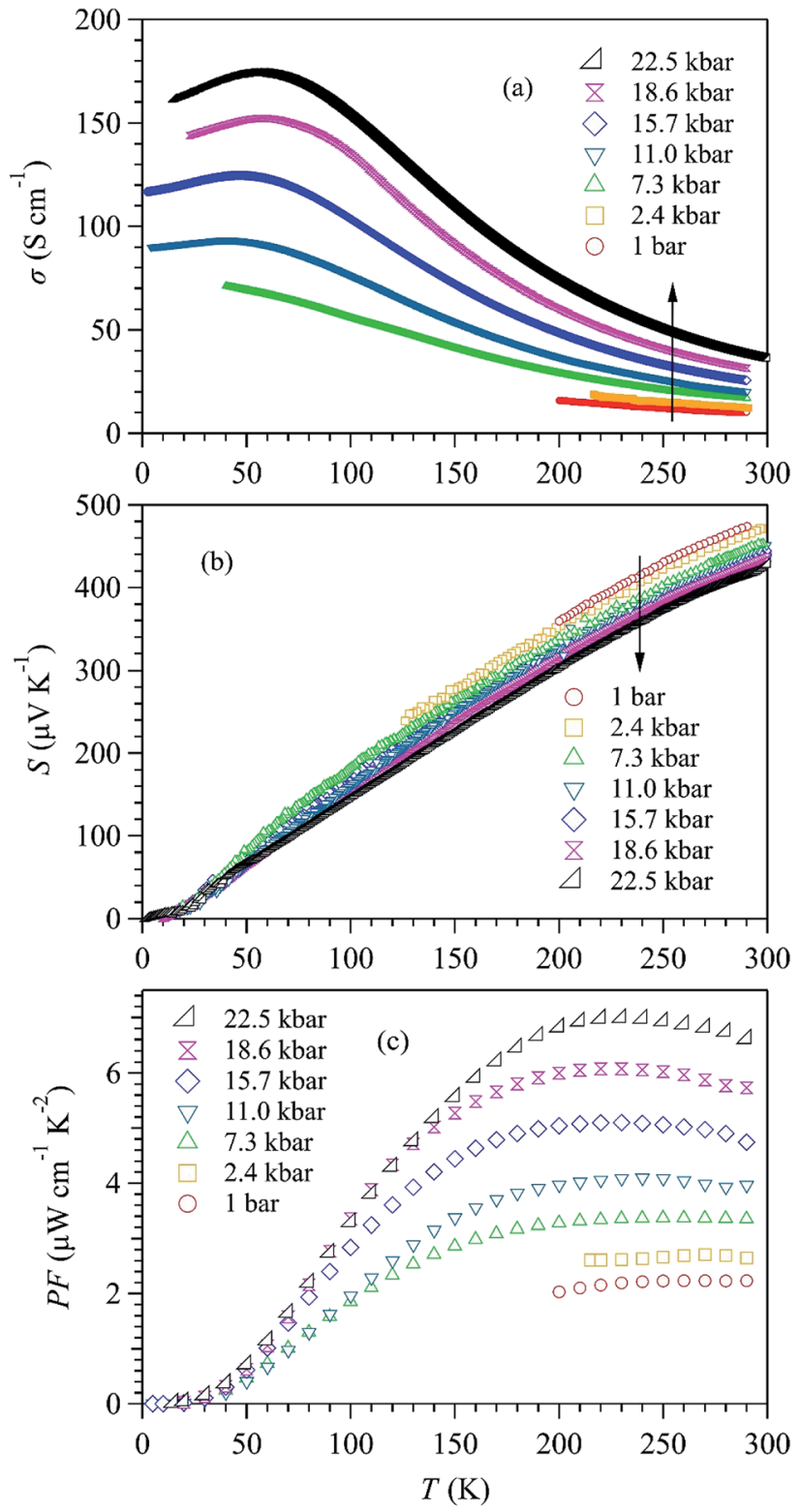

Fig. 3 Temperature dependence of (a) electrical conductivity $\sigma(T)$, (b) Seebeck coefficient $S(T)$, and (c) power factor PF $\equiv S^{2} \sigma$ of pristine SnSe crystal under various pressures up to 22.5 kbar.

\section{Results and discussion}

\section{Pressure-induced enhancement of PF in pristine SnSe}

Fig. 3(a) shows the $\sigma(T)$ of pristine SnSe below $300 \mathrm{~K}$ under various pressures up to $22.5 \mathrm{kbar}$. For our studied sample, the room-temperature $\sigma$ at 1 bar is about $10 \mathrm{~S} \mathrm{~cm}^{-1}$, which is close to that reported previously. ${ }^{5}$ Upon cooling down, $\sigma(T)$ increases gradually, and exhibits a metallic rather than an intrinsic semiconducting behavior. This is caused by a slight hole doping due to the presence of Sn vacancies as verified by the EDS measurements, Fig. 1(c). As shown in Fig. 3(a), the measurements of $\sigma(T)$ at the first two pressures were terminated at $\sim 200$ $\mathrm{K}$ because the contact resistance became too large to get reliable data at low temperatures. This issue was overcome at higher pressures when the electrical contacts are improved. As can be seen, the application of high pressure boosts the $\sigma(T)$ of pristine SnSe in the whole temperature range; the room-temperature $\sigma$ increases from $\sim 10 \mathrm{~S} \mathrm{~cm}^{-1}$ at 1 bar to $\sim 38 \mathrm{~S} \mathrm{~cm}^{-1}$ at $22 \mathrm{kbar}$, also shown in Fig. 5(a), which is nearly four-fold enhancement. The $\sigma(T)$ at high pressures retains a metallic behavior over a wide temperature range from $300 \mathrm{~K}$ down to $\sim 50-70 \mathrm{~K}$, below which a shallow downturn appears. The maximum conductivity at low temperatures can reach $\sim 170 \mathrm{~S} \mathrm{~cm}^{-1}$ at $22.5 \mathrm{kbar}$. According to the band-structure calculations under pressure, ${ }^{\mathbf{1 8 , 2 0}}$ the essential features of most bands keep intact in the investigated pressure range, but pressure-induced reduction of band gap should be responsible for the observed enhancement of electrical conductivity.

The increase of $\sigma(T)$ should usually be accompanied by a decrease of $S(T)$, but the PF depends on the relative change of these two anti-correlated parameters. As shown in Fig. 3(b), for all pressures $S(T)$ increases with temperature, consistent with the metallic behavior of $\sigma(T)$ shown in Fig. 3(a). As expected, $S(T)$ in the whole temperature range is indeed reduced gradually upon applying pressure, but the reduction is rather moderate. The $S$ value at room temperature decreases by only $\sim 13 \%$ from $\sim 480$ $\mu \mathrm{V} \mathrm{K} \mathrm{K}^{-1}$ at 1 bar to $\sim 420 \mu \mathrm{V} \mathrm{K}^{-1}$ at $22.5 \mathrm{kbar}$, also shown in Fig. 3(b). Such a moderate change of $S(T)$ is in sharp contrast to the strong enhancement of $\sigma(T)$ shown in Fig. 3(a). The robustness of $S(T)$ against pressure indicates that the unique multiple valley valence band structure is preserved under pressure.

The strong enhancement of $\sigma(T)$ accompanied with a moderate reduction of $S(T)$ under pressure gives rise to a strong boost of PF for pristine SnSe, as illustrated in Fig. 3(c). In addition, $\mathrm{PF}(T)$ exhibits a broad plateau and almost parallel enhancement over a wide temperature range between 150 and $300 \mathrm{~K}$. The PF of SnSe at $290 \mathrm{~K}$ increases by 3 times from $\sim 2.24$ $\mu \mathrm{W} \mathrm{cm} \mathrm{cm}^{-1} \mathrm{~K}^{-2}$ at 1 bar to $6.61 \mu \mathrm{W} \mathrm{cm}{ }^{-1} \mathrm{~K}^{-2}$ at $22.5 \mathrm{kbar}$. As shown in Fig. 5(c), the PF (290 K) increases almost linearly with pressure, without showing any saturation up to $22.5 \mathrm{kbar}$. As a matter of fact, the PF $(290 \mathrm{~K})$ at $22.5 \mathrm{kbar}$ is close to the optimal PF values in the high- $T$ Cmcm phase at ambient pressure. ${ }^{5,9}$ Our results thus confirmed the theoretical prediction that pressure can intrinsically enhance the TE properties of SnSe over a wide temperature region in the low- $T$ Pnma phase. ${ }^{18}$

\section{Pressure-induced enhancement of PF in Na-doped SnSe}

To check if the high-pressure approach is universal for the SnSebased TE materials, we performed similar measurements on Na-doped SnSe up to $19.2 \mathrm{kbar}$. The results are displayed in Fig. $4(\mathrm{a}-\mathrm{c})$. Because the carrier concentration of $\mathrm{Na}-\mathrm{SnSe}$ is about two orders higher than that of the pristine sample, ${ }^{6}$ the Na-doped SnSe exhibits a much larger $\sigma \sim 830 \mathrm{~S} \mathrm{~cm}^{-1}$ yet a lower $S \sim 200 \mu \mathrm{V} \mathrm{K}^{-1}$ at room temperature. Similar with those of pristine SnSe, the $\sigma(T)$ of Na-SnSe also increases while $S(T)$ decreases upon applying pressure, as shown in Fig. 4(a) and (b). However, their relative changes under pressure are different from those of pristine SnSe. As illustrated in Fig. 5(a) and (b), when increasing pressure from 1 bar to $19.2 \mathrm{kbar}$ on the $\mathrm{Na}-$ doped SnSe at room temperature, its $\sigma(290 \mathrm{~K})$ increases by $\sim 3$ times from 830 to $2445 \mathrm{~S} \mathrm{~cm}^{-1}$, while the $S$ decreases by $\sim 30 \%$ 

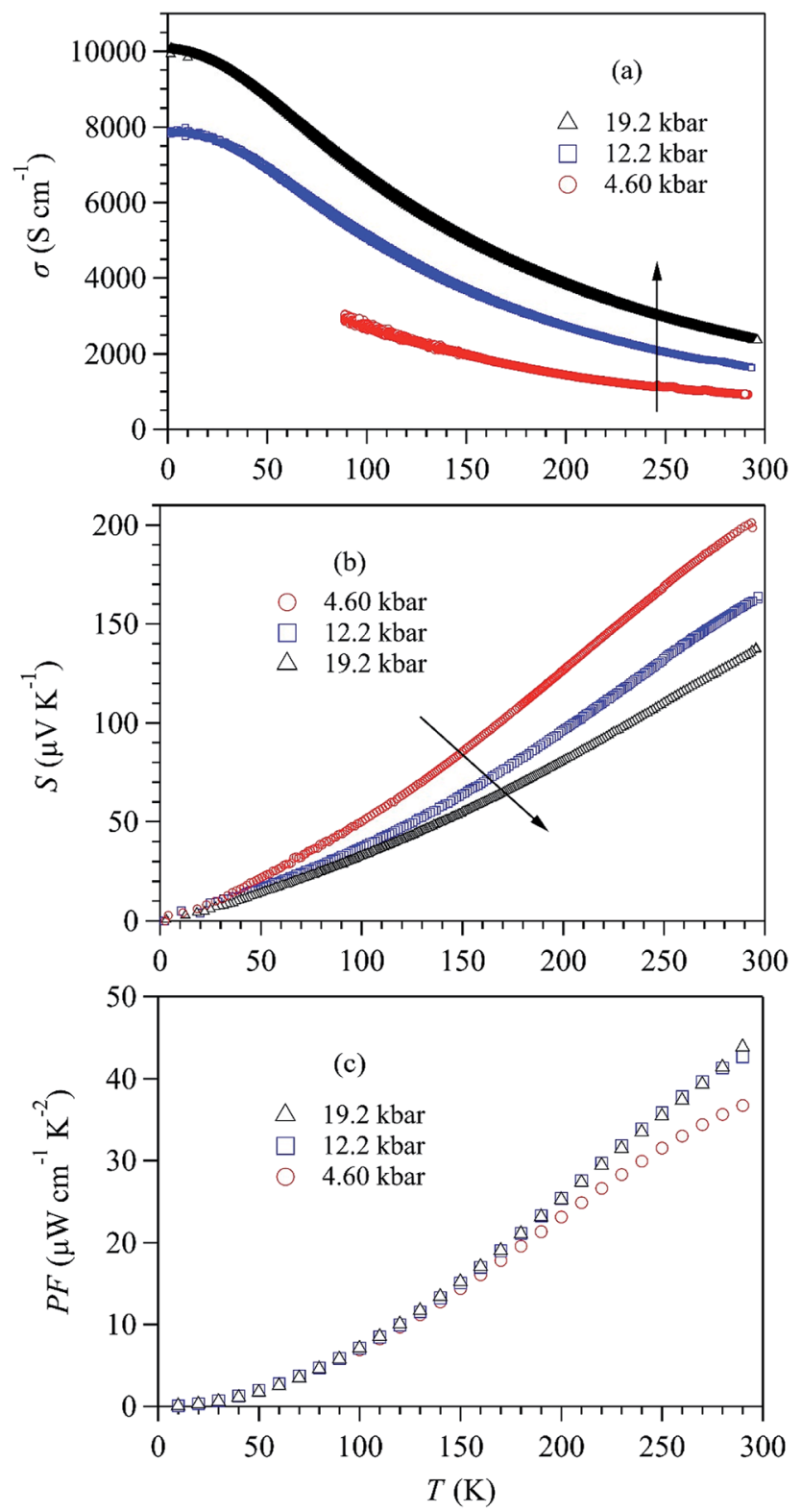

Fig. 4 Temperature dependence of (a) electrical conductivity $\sigma(T)$, (b) Seebeck coefficient $S(T)$, and (c) power factor PF of Na-doped SnSe crystal under various pressures up to 19.2 kbar.

from $\sim 200$ to $134 \mu \mathrm{V} \mathrm{K}^{-1}$. As a result, the enhancement of PF is marginal as illustrated in Fig. 4(c). In addition, the PF enhancement appears mainly in the low-pressure region near room temperature. The PF at $\mathbf{1 2 . 2}$ kbar almost overlaps with that at 19.2 kbar. As shown in Fig. 5(c), PF (290 K) increases from $33.2 \mu \mathrm{W} \mathrm{cm}{ }^{-1} \mathrm{~K}^{-2}$ at 1 bar to $43.9 \mu \mathrm{W} \mathrm{cm}{ }^{-1} \mathrm{~K}^{-2}$ at 19.25 kbar. In contrast to the linear increase with pressure for pristine SnSe, the PF of Na-SnSe tends to level off at higher pressures. These comparison thus underscores a peculiar pressure effect on the enhancement of PF in pristine SnSe.

\section{Pressure-induced reduction of PF in black phosphorus}

To further substantiate the underlying mechanism, a comparison with the pressure effect on black phosphorus (BP) is

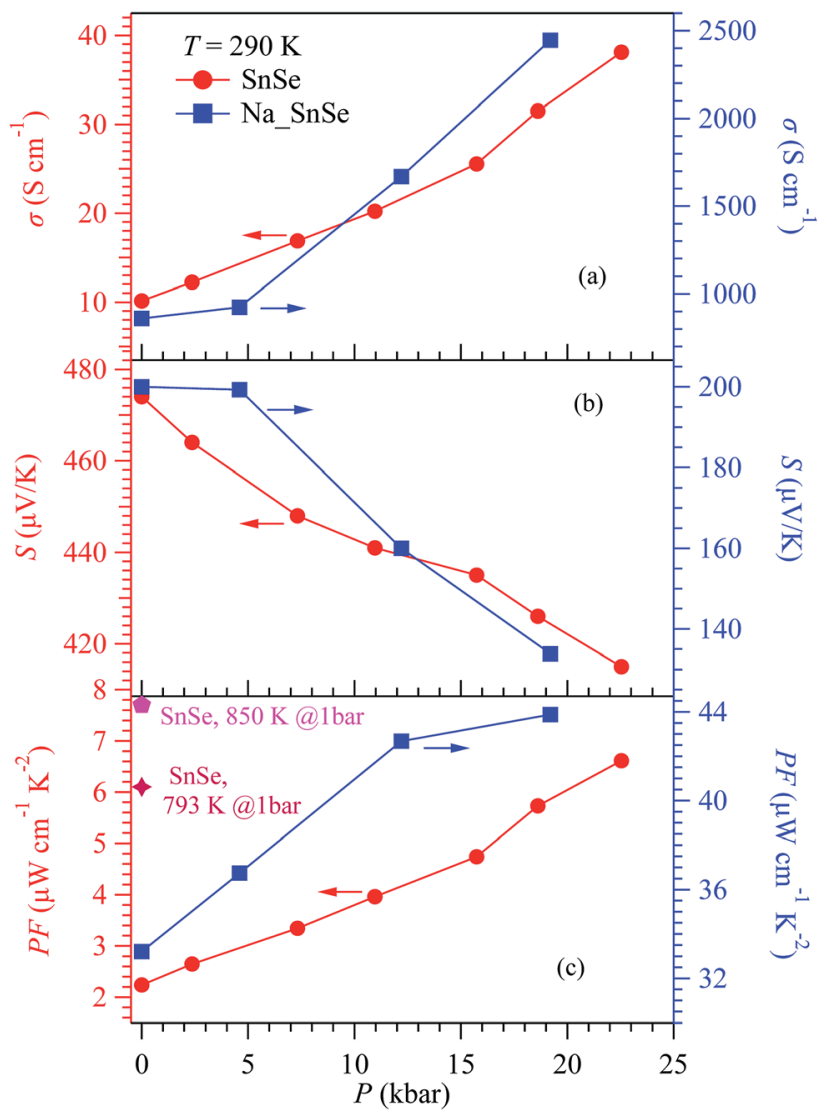

Fig. 5 Pressure dependence of (a) conductivity $\sigma$, (b) Seebeck coefficient S, and (c) power factor PF at $290 \mathrm{~K}$ for pristine SnSe (left) and $\mathrm{Na}$-doped SnSe (right). The PF values of SnSe at high temperatures and ambient pressure (pentagon and star symbols) in (c) are taken from ref. 5 and 9 , respectively.

instructive, because it has a similar crystal structure as SnSe but much simpler band structure. Bulk BP is a narrow gap semiconductor with a direct energy gap $\sim 0.33 \mathrm{eV}$ at $Z$ point of Brillouin zone. ${ }^{21,22}$ Previous high-pressure studies have demonstrated a pressure-induced Lifshitz transition at about 12 kbar associated with the closure of the direct band gap. ${ }^{23}$ This leads to a significant enhancement of conductivity. As illustrated in Fig. 6(a), $\sigma(290 \mathrm{~K})$ of BP increases by 36 times from $\sim 4.4 \mathrm{~S} \mathrm{~cm}^{-1}$ at 1 bar to $\sim 160 \mathrm{~S} \mathrm{~cm}^{-1}$ at $20 \mathrm{kbar}$. The enhancement of conductivity in BP is much stronger than that seen in SnSe, Fig. 5(a), because the larger band gap of SnSe $\sim 0.6-0.8 \mathrm{eV}$ cannot be closed by the applied pressure of $\sim 22$ kbar. ${ }^{18}$ In the semimetal state of BP above 12 kbar, large positive magnetoresistance and Shubnikov-de Haas oscillations have been detected. ${ }^{23}$ In our recent study, ${ }^{24}$ we measured $S(T)$ of BP under pressure and also evidenced the closure of the direct band gap at 10-12 kbar, manifested by the vanishing $S(T)$ hump and the change of $\mathrm{d} S / \mathrm{d} T$ near room temperature from negative to positive. As a result, the $S(290 \mathrm{~K})$ decreases by an order of magnitude from $\sim 260 \mu \mathrm{V} \mathrm{K}^{-1}$ at 1 bar to $\sim 23 \mu \mathrm{V} \mathrm{K} \mathrm{K}^{-1}$ at $20 \mathrm{kbar}$, as shown in Fig. 6(b). Then, the PF of BP can be calculated and is displayed in Fig. 6(c) as a function of pressure. Apparently, the $\mathrm{PF}$ experiences a strong reduction across the Lifshitz transition when the direct band gap is closed by pressure. It is 


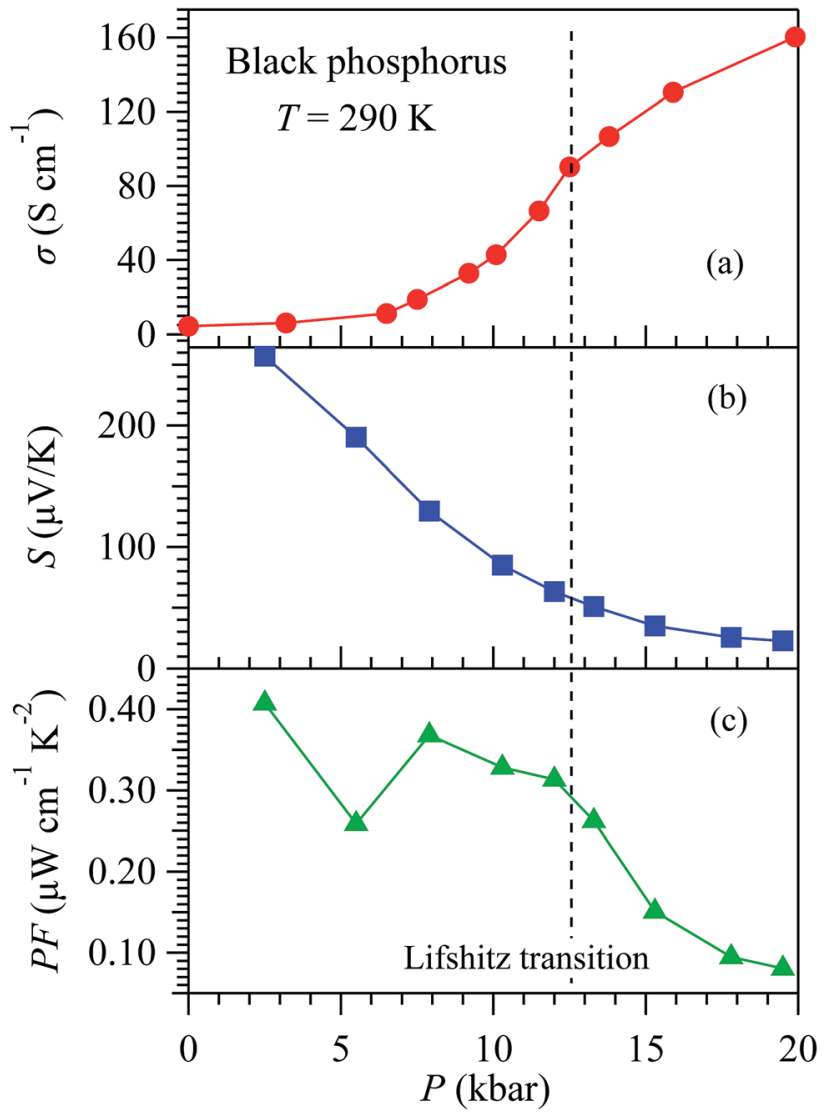

Fig. 6 Pressure dependence of (a) electrical conductivity $\sigma$, (b) Seebeck coefficient $S$, and (c) power factor PF at $290 \mathrm{~K}$ for black phosphorus. The Lifshitz transition taking place around $12 \mathrm{kbar}$ is marked by the dotted vertical line.

noteworthy that the PF of BP also decreases gradually in the lowpressure region before closing the direct band gap. This fact demonstrates that the reduction of band gap in a simple semiconductor that enhances $\sigma$ while reduces $S$ simultaneously cannot guarantee a boost of PF. This is true in general because the enhancement of $\sigma$ is usually overcome by the reduction of $S^{2}$.

\section{Discussions}

The distinct pressure effects on PF between SnSe and BP thus suggest that we cannot simply attribute the three-fold enhancement of PF in SnSe to the reduction of band gap ${ }^{18}$ or a Lifshitz transition. ${ }^{25}$ Instead, it should have a deep root on the unique band structure of SnSe. As shown by the DFT calculations ${ }^{18}$ and APRES experiments, ${ }^{26}$ SnSe in the Pnma phase has complex electronic valence bands, which are featured by multiple valence band maxima within a small energy range. The small energy gaps between these valence band maxima can be crossed by the Fermi level via hole doping. The experimental Seebeck coefficient at ambient pressure can only be reproduced by considering the multi-valley band structures. ${ }^{6}$ In contrast, a single band model cannot reproduce the experimental Seebeck coefficient even considering a large band effective mass enhancement. Thus, the unique electronic band structure with multiple valence band maxima lying close in energy is the key to realize enhanced Seebeck coefficient and PF at ambient pressure. If this feature is preserved under a moderate pressure, the Seebeck coefficient can retain a large value and thus ensure an enhanced PF under pressure.

\section{Conclusion}

In summary, we have studied the pressure effect on the TE properties of pristine and holed-doped SnSe crystals. Our results reveal a three-fold enhancement of PF for pristine SnSe under a pressure to $22 \mathrm{kbar}$ without showing a trend of saturation at higher pressures. We also observe a moderate enhancement of PF for Na-doped SnSe under pressure. In comparison with the monotonic reduction of $\mathrm{PF}$ in black phosphorus under pressure, our results underscore the importance of the unique electronic band structure of SnSe in producing the high Seebeck coefficient. The presence of multiple valence band maxima that preserves under pressure should play an important role in retaining a large Seebeck coefficient and thus an enhanced power factor by the application of high pressure.

\section{Author contributions}

J. G. C. conceived and supervised this project. B. C. Q., K. J. Z., Y. G. S. and L. D. Z. prepared the single-crystal samples. N. S., Z. Y. L., P. S., J. P. S., B. S. W, and Y. S. carried out the measurements of thermoelectric properties under high pressure. N. S. and J. G. C. performed the data analysis and wrote the paper. All authors discussed the results and commented on the manuscript.

\section{Conflicts of interest}

There are no conflicts to declare.

\section{Acknowledgements}

This work is supported by the National Key R\&D Program of China (Grant No. 2018YFA0305700, 2018YFA0305800, and 2017YFA0302901), the National Natural Science Foundation of China (Grant No. 11574377, 11834016, 11874400, 11774399), the Strategic Priority Research Program and Key Research Program of Frontier Sciences of the Chinese Academy of Sciences (Grant No. XDB07020100 and Grant No. QYZDB-SSWSLH013) as well as the CAS Interdisciplinary Innovation Team. J. P. S. acknowledges support from the Postdoctoral Innovative Talent program. We thank Dr Laiquan Shen and Prof. Yanhui Liu for the SEM measurements.

\section{References}

1 J. He and T. M. Tritt, Science, 2017, 357, eaak9997.

2 Z.-G. Chen, G. Han, L. Yang, L. Cheng and J. Zou, Prog. Nat. Sci.: Mater. Int., 2012, 22, 535. 
3 J. P. Heremans, M. S. Dresselhaus, L. E. Bell and D. T. Morelli, Nat. Nanotechnol., 2013, 8, 471.

4 K. Zhao, H. Duan, N. Raghavendra, P. Qiu, Y. Zeng, W. Zhang, J. Yang, X. Shi and L. Chen, Adv. Mater., 2017, 29, 1701148.

5 L. D. Zhao, S. H. Lo, Y. S. Zhang, H. Sun, G. J. Tan, C. Uher, C. Wolverton, V. P. Dravid and M. G. Kanatzidis, Nature, 2014, 508, 373.

6 L. D. Zhao, et al., Science, 2016, 351, 141.

7 C. L. Chen, H. Wang, Y. Y. Chen, T. Day and G. J. Snyder, J. Mater. Chem. A, 2014, 2, 11171.

8 E. K. Chere, Q. Zhang, K. Dahal, F. Cao, J. Mao and Z. F. Ren, J. Mater. Chem. A, 2016, 4, 1848.

9 M. Jin, et al., ACS Appl. Mater. Interfaces, 2019, 11, 8051.

10 H. S. Kim, G. Choi, M. Y. Ha, D. H. Kim, S. H. Park, I. Chung and W. B. Lee, J. Solid State Chem., 2019, 270, 413.

11 V. Kucek, T. Plechacek, P. Janicek, P. Ruleova, L. Benes, J. Navratil and C. Drasar, J. Electron. Mater., 2016, 45, 2943.

12 J. R. Li, J. T. Xu, H. X. Wang, G. Q. Liu, X. J. Tan, H. Z. Shao, H. Y. Hu and J. Jiang, J. Mater. Sci.: Mater. Electron., 2018, 29, 18727.
13 C. C. Lin, D. Ginting, G. Kim, K. Ahn and J. S. Rhyee, Curr. Appl. Phys., 2018, 18, 1534.

14 X. L. Shi, et al., Adv. Eng. Mater., 2019, 9, 1803242.

15 X. l. Shi, A. y. Wu, W. Liu, R. Moshwan, Y. Wang, Z. G. Chen and J. Zou, ACS Nano, 2018, 12, 11417.

16 P. C. Wei, et al., ACS Omega, 2019, 4, 5442.

17 B. Qin, D. Wang, W. He, Y. Zhang, H. Wu, S. J. Pennycook and L.-D. Zhao, J. Am. Chem. Soc., 2019, 141, 1141.

18 Y. S. Zhang, S. Q. Hao, L. D. Zhao, C. Wolverton and Z. Zeng, J. Mater. Chem. A, 2016, 4, 12073.

19 P. Shahi, et al., Phys. Rev. X, 2018, 8, 021055.

20 J. J. Yan, et al., Phys. Chem. Chem. Phys., 2016, 18, 5012.

21 Y. Takao and A. Morita, Physica B+C, 1981, 105, 93.

22 Y. Takao, H. Asahina and A. Morita, J. Phys. Soc. Jpn., 1981, 50, 3362.

23 Z. J. Xiang, et al., Phys. Rev. Lett., 2015, 115, 186403.

24 X. Li, et al., Proc. Natl. Acad. Sci. U. S. A., 2018, 115, 9935.

25 T. Nishimura, et al., Phys. Rev. Lett., 2019, 122, 226601.

26 I. Pletikosić, F. Von Rohr, P. Pervan, P. K. Das, I. Vobornik, R. J. Cava and T. Valla, Phys. Rev. Lett., 2018, 120, 156403. 